\title{
Geometry of the Hilbert space and the Quantum Zeno Effect
}

\author{
A. K. Pati and S. V. Lawande \\ Theoretical Physics Division, 5th Floor, Central Complex, \\ Bhabha Atomic Research Centre, Mumbai - 400 085, INDIA.
}

(July 27, 2021)

\begin{abstract}
We show that the quadratic short time behaviour of transition probability is a natural consequence of the inner product of the Hilbert space of the quantum system. We provide a relation between the survival probability and the underlying geometric structure such as the Fubini-Study metric defined on the projective Hilbert space of the quantum system. This predicts the quantum Zeno effect even for systems described by non-linear and non-unitary evolution equations, within the collapse mechanism of the wavefunction during measurement process.
\end{abstract}

PACS NO: 03.65.Bz

email: krsrini@magnum.barc.ernet.in 
Using collapse postulate of quantum measurement theory Mishra and Sudarshan [1] have shown that the life-time of an unstable system can be prolonged by performing "frequent observations". In the limit of infinitely frequent observations the decay is inhibited, which is referred to as the quantum Zeno paradox (QZP). Similarly, for stable systems one can prove that if we prepare the system in any one of the eigenstates of the observable and interrupt the unitary evolution of the system in succession, the transition to other eigenstate is prohibited. The inhibition of transition for coherently evolving system under continuous observation is often termed as quantum Zeno effect (QZE) [2]. These predictions are based on principles of linear quantum theory and on the projection postulates of von Neumann [3] which results in short time behaviour of the transition probability that grows quadratically with time. In general, the state of a system evolves unitarily according to Schrödinger equation and to obtain a result on some observable we need to do a measurement. During measurement some coupling occurs between the system and the measuring apparatus and the apparatus reads one of the eigenvalues of the observable of the system. The state of the system which is in a superposition of different eigenstates corresponding to different eigenvalues of the observable, collapses to that eigenstate corresponding to the eigenvalue being measured. This constitutes a precise and instantaneous measurement.

Although the original formulation of QZP has not been tested experimentally, the QZE for a stable system is claimed to have been observed by Itano et al [4]. Following Cook's [5] proposal Itano's group made use of a two-level atom coupled to a third level via optical pulses so as to monitor the atom in its ground state. Two explain their observations they use the two ingredients, namely, (i) the Schrödinger time evolution between two successive measurements and (ii) the reduction mechanism and their results were found to be in full agreement with the theoretical predictions. In a sense this interpretation was considered to provide an experimental support of the collapse postulate and QZE. Subsequently, it was argued by many physicists [6, [7] that the experiment of Itano et al [4] in no way verifies von Neumann's collapse postulate. It was also shown [6:7] that the results of Itano et al [4] could be explained by making use of unitary dynamical evolution alone and the collapse postulate was, in fact, not necessary.

Recently, there have been some arguments to clarify the meaning of continuous observation within the conventional quantum theory. The continuous observation or measurement is understood as the limit of a sequence of discrete, instantaneous ones when the interval between two successive measurements tends to zero (which amounts to taking $\mathrm{N}$, the number of measurements to infinity). Sometimes back Ghiradi et al [8] have argued that the complete suppresion of decay of an unstable system cannot be realised due to time-energy uncertainty relation in the original context of QZP. Nakazato et al [9] have argued that infinite limit of frequent measurement cannot be taken due to losses in the real experimental setups and also due to spreads in the position and momentum of a quantum particle. 
Also an approach based on decoherence [10] indicates that the finite decoherence time sets a limit on the interval between two successive measurements. Using the geometric ideas of quantum state space and the generalised uncertainty relation one of the present authors [11] has shown that there is a limit on the frequency of measurement in quantum Zeno setups and the previously discussed limits are special cases of this generalised limit.

In past several years, there have been different approaches suggested to replace von Neumann's collapse postulate [12]. Most interestingly, the QZE has been understood using a continuous measurement model based on restricted Feynman path integrals [13]. This results in an effective Hamiltonian description that is non-hermitian in nature. The imaginary part of the Hamiltonian takes into account the effect of meter on measured system. There has been a stochastic simulation of quantum trajectory approach to understand the QZE for ensemble of ions [14]. Here, the inhibition of transition is achieved by destroying the coherence. Since repeated measurements on quantum systems have considerable interest in a variety of situations [15], it would be important to relax some of the basic postulates of quantum theory so that the quantum Zeno effect may be realised in wide class of systems. Recently, a nice review of theoretical and experimental status of the quantum Zeno effect has been given by Home and Whitaker [16].

In this letter we examine the necessity of the Schrödinger quantum evolution between two successive measurements. We recall that the usual proof of QZE, is based on two basic postulates of quantum theory, viz, (i) the state of the system evolves according to Schrödinger equation between $[0, \tau],[\tau, 2 \tau], \ldots \ldots .[(N-1) \tau, N \tau]$ and (ii) the measurements are performed instantaneously at discrete times $\tau, 2 \tau, \ldots . . N \tau$, such that total duration of the process $T=N \tau$ is held fixed. As pointed out above the necessity of the collapse postulate has been questioned and it has been shown that a pure dynamical equation could predict the QZE. Here, we question the requirement of the first postulate, namely, the linear unitary time evolution of the quantum system between two successive measurements. We argue that it is not a necessary but only a sufficient requirement for predicting QZE. All we need is that the soultion of the evolution equation should belong to a Hilbert space. We argue that the inner product defined on the Hilbert gives us the quadratic short-time behaviour, which is necessary for the occurence of QZE, within the collapse postulate. It is well known from the study of geometry of quantum evolution [17 20] that the inner product also induces the metric structure on the projective Hilbert space of the quantum system. We show that the survival probability is directly related to this geometric structure of the Hilbert space. To define the metric structure the state vector need not follow a Schrödinger time evolution. Further, we provide a criterion for observing the QZE for a finite number of measurements as an infinite number of frequent measurements is not possible within the von Neumann type of measurement scheme. As a consequence of the geometry of the Hilbert space, one can observe QZE for systems described by non-linear Schrödinger equation [20] and Gisin's 
equation [21]. These equations give rise to a quadratic short-time behaviour for transition probabilities. The prediction of QZE for systems governed by non-linear equations is rather interesting because in the quadratic time-dependence regime the behaviour of transition probablity can depend on the non-linearity parameter of the dynamical system. This may accelerate the inhibition process under repeated observation as compared to that expected from a linear dynamical evolution process.

We consider a quantum system whose state vector $\mid \Psi(t)>\in \mathcal{H}$, where $\mathcal{H}$ is the Hilbert space of the system with dimension $\mathrm{K}$, i.e., $\mathcal{H}=C^{K}$. We are interested in measuring some observable $O$ of the system, which is represented by a Hermitian operator. It has an eigenvalue spectrum $\left\{O_{n}\right\}$ and a complete set of eigenfunctions $\left\{\mid \Psi_{n}>\right\}$. The spectrum is assumed to be discrete and non-degenerate. Let the system be prepared initially in the state $\mid \Psi(0)>$ which could be an eigenfunction of the observable $O$. Without loss of generality we can assume that the initial state is normalised to unity. However, at a later time the normalisation of the state vector is not guarranted. Now, we allow the system to evolve under some dynamical law and ask the question: How does the probability of finding the system in the initial state at a later time $\tau$ behaves with $\tau$, where $\tau$ is assumed to be small. We do not assume the unitary, linear Schrödinger quantum evolution to be satisfied by the state vector $\mid \Psi(t)>$ in the interval $[0, \tau]$. The survival probability for the system to be in the initial state is defined as

$$
P(\tau)=\left|\left\langle\frac{\Psi(0)}{\|\Psi(0)\|} \mid \frac{\Psi(\tau)}{\|\Psi(\tau)\|}\right\rangle\right|^{2},
$$

where $\|\Psi(t)\|=<\Psi(t) \mid \Psi(t)>^{\frac{1}{2}}$ denotes the norm of the state vector defined from the inner product.

We introduce the normalised vector $|\chi(t)>=| \frac{\Psi(t)}{\|\Psi(t)\|}>$. On Taylor expanding $\mid \chi(\tau)>$ arround $t=0$, we obtain

$$
|\chi(\tau)>=| \chi(0)>+\tau\left|\dot{\chi}(0)>+\frac{\tau^{2}}{2}\right| \ddot{\chi}(0)>+O\left(\tau^{3}\right) .
$$

Therfore, the survival amplitude of finding the system in the initial state for short time is given by

$$
<\chi(0)|\chi(\tau)>=1+\tau<\chi(0)| \dot{\chi}(0)>+\frac{\tau^{2}}{2}<\chi(0) \mid \ddot{\chi}(0)>+O\left(\tau^{3}\right) .
$$

Hence, the probability of finding the system at time $\tau$ in the initial state (which is called survival probability) is given by

$$
P(\tau)=1+\tau^{2}\left[\operatorname{Re}<\chi(0) \mid \ddot{\chi}(0)>+(\operatorname{Im}<\chi(0) \mid \dot{\chi}(0)>)^{2}\right] .
$$

Since $\mid \chi(t)>$ preserves the norm $\|\chi(t)\|$ during the time evolution, one can show that the quantity $<\chi(t) \mid \dot{\chi}(t)>$ is purely imaginary and $R e<\chi(t)|\ddot{\chi}(t)>=-<\dot{\chi}(t)| \dot{\chi}(t)>$. Using these facts, we can express the survival probability as 


$$
\begin{aligned}
P(\tau) & \left.=1-\tau^{2}[<\dot{\chi}(0) \mid \dot{\chi}(0)>)-(i<\chi(0) \mid \dot{\chi}(0)>)^{2}\right] \\
& =1-\tau^{2} k
\end{aligned}
$$

where $\left.k=[<\dot{\chi}(0) \mid \dot{\chi}(0)>)-(i<\chi(0) \mid \dot{\chi}(0)>)^{2}\right]$.

The quantity $k$ can also be expressed in terms of the actual state (unnormalised state vector $|\Psi\rangle$ ) of the system. Notice that we can write

$$
<\dot{\chi}(0)\left|\dot{\chi}(0)>=\frac{<\dot{\Psi}(0) \mid \dot{\Psi}(0)>}{\|\Psi(0)\|^{2}}+\frac{\|\dot{\Psi}(0)\|^{2}}{\|\Psi(0)\|}-\frac{\|\dot{\Psi}(0)\|}{\|\Psi(0)\|^{3}} 2 R e<\Psi(0)\right| \dot{\Psi}(0)>
$$

and

$$
(i<\chi(0) \mid \dot{\chi}(0)>)^{2}=\frac{|<\Psi(0)| \dot{\Psi}(0)>\left.\right|^{2}}{\|\Psi(0)\|^{4}}+\frac{\|\dot{\Psi}(0)\|^{2}}{\|\Psi(0)\|}-\frac{\|\dot{\Psi}(0)\|}{\|\Psi(0)\|^{3}} 2 R e<\Psi(0) \mid \dot{\Psi}(0)>
$$

Using above expressions we can write the constant $k=\left[\frac{<\dot{\Psi}(0) \mid \dot{\Psi}(0)>}{\|\Psi(0)\|^{2}}-\frac{|<\Psi(0)| \dot{\Psi}(0)>\left.\right|^{2}}{\|\Psi(0)\|^{4}}\right]$.

Now, the next step would be to argue that the constant $k$ is a non-negative (whose physical meaning will be discussed). Note that the transition probability of finding the system in the initial state has $\tau^{2}$ dependence, for short times, which comes naturally from the inner product of the vectors defined over the Hilbert space of the quantum system. In order that the coherent time evolution leads to a survival probability approaching unity under repeated observation the non-negativity of $k$ is crucial. In the following we provide an argument based on Hilbert space geometry that it is indeed so.

To provide a physical meaning to the quantity $k$ in a general situation, we take recourse to some basic ideas of geometry of quantum evolution. Quantum mechanically, the system is represented not just by a vector but by a ray. A ray is a set of vectors which differ from each other by $U(1)$ phase factors. If we take a projection $\Pi: \mathcal{H} \rightarrow \mathcal{P}$, then all the points in a ray project to a single point in the projective Hilbert space of the quantum system. Therefore, geometrically the system is represented by a point on the projective Hilbert space $\mathcal{P}$ of the quantum system. The projective Hilbert space is formed by taking the set of rays of the Hilbert space. Let $\{\mid \Psi>\} \in \mathcal{H}$, then consider the set of non-zero vectors of unit norm $\left\{\mid \chi>=\frac{\mid \Psi>}{\|\Psi\|}\right\} \in \mathcal{H}^{*}$. Thus, the projective Hilbert space $\mathcal{P}=\mathcal{H}^{*} / U(1)$, where $\mathrm{U}(1)$ is the group with non-zero complex numbers. The evolution of the system is represented by a curve in $\mathcal{P}$ and if we want to know how much distance has been travelled by the system point $\Pi(\mid \chi>)$, then we need to know the metric defined on it.

Some times back it has been shown by Provost and Vallee in [17 that the inner product in the Hilbert space can induce a Riemannian structure on the projective Hilbert of the quantum system. In studying geometry of quantum evolution and geometric phases the inner product between two state vectors once again plays a very important role. The inner 
product or survival amplitude between two non-orthogonal states is in general complex number, which has a magnitude and a phase. The physical meaning of the magnitude is that its square gives us transition probability (which is of concern here). The phase in general contains a dynamical and a geometric component. The geometric phase is one of the fundamental discovery in recent times which tells us that the wavefunction of a quantum system can acquire a phase depending solely on the geometry of the path in the projective Hilbert space of the system [23]. It is worth mentioning that quantum Zeno effect does not answer the question: what happens to phase under repeated measurements? This is an important question which was addressed only recently by the present authors [24]. The quest of geometric structures led Aharonov and Anandan [18] to introduce Fubini-Study metric which provides the total distance travelled by the system point along the evolution curve in the projective Hilbert space of quantum system. The connection between geometric phases and the metric structures were clarified by one of the present authors [19]. With the help of the metric structures many quantum mechanical phenomena has been viewed in a geometric way. Further, the Fubini-Study metric was generalised by one of the present authors 20 to case of non-unitary, non-Schrödinger evolutions arising from the inner product of vectors, which is given by

$$
s^{2}=4\left(1-\left|\left\langle\frac{\Psi_{1}}{\left\|\Psi_{1}\right\|} \mid \frac{\Psi_{2}}{\left\|\Psi_{2}\right\|}\right\rangle\right|^{2}\right)
$$

Physically, this metric is a measure of the distance between two states in the Hilbert space or the corresponding points in the projective Hilbert space of the quantum system. It satisfies identity, symmetry, and triangle inequality conditions. It is invariant under $U(1)$ gauge transformation as well as under generalised gauge transformation (see [20]). When the two vectors differ infinitesimally, we obtain the infinitesimal Fubini-Study metric defined as

$$
d s^{2}=4\left[\left\langle\frac{d}{d t}\left(\frac{\Psi(t)}{\|\Psi(t)\|}\right) \mid \frac{d}{d t}\left(\frac{\Psi(t)}{\|\Psi(t)\|}\right)\right\rangle-\left(i\left\langle\frac{\Psi(t)}{\|\Psi(t)\|} \mid \frac{d}{d t}\left(\frac{\Psi(t)}{\|\Psi(t)\|}\right)\right\rangle\right)^{2}\right] d t^{2} .
$$

This generalised metric [20] is valid even when a system undergoes non-linear, nonunitary and inhomogeneous evolution equation. In the special case of linear, unitary evolution this reduces to the Fubini-Study metric defined in [18,19. This metric is invariant under gauge transformation $\left|\Psi(t)>\rightarrow e^{i \alpha(t)}\right| \Psi(t)>$. Also, it is invariant under generalised gauge transformation $|\Psi(t)>\rightarrow Z(t)| \Psi(t)>$, with $Z(t)$ being a complex function of nonunit modulus. Since the modulus of the inner product is invariant under all unitary and anti-unitary transformations, the metric is so. Moreover, this does not depend on the detailed dynamics of the system. If we define the speed at which the system point moves on the projective Hilbert space of the quantum system is $v(t)=d s / d t$, then the total distance travelled by the system point during an arbitrary quantum evolution is given by

$$
s=\int v(t) d t
$$


where $\left.v(t)=2[<\dot{\chi}(t) \mid \dot{\chi}(t)>)-(i<\chi(t) \mid \dot{\chi}(t)>)^{2}\right]^{\frac{1}{2}}$, which can also be written as $v=$ $2\left[\frac{\langle\dot{\Psi}(t)| \dot{\Psi}(t)>}{\|\Psi(t)\|^{2}}-\frac{|<\Psi(t)| \dot{\Psi}(t)>\left.\right|^{2}}{\|\Psi(t)\|^{4}}\right]^{\frac{1}{2}}$. This geometric quantity is a reprametrisation invariant and depends only on the projected path of the evolving quantum system.

It follows that the quantity $k$ appearing in the survival probability (5) has a clear physical meaning of being the square of the the speed of transportation of the system point on $\mathcal{P}$ evaluated at initial time i.e., $k=v^{2}(0) / 4$. The non-negativity of the real constant $k$ is therefore guaranted.

With this geometrical interpretation of survival probability $P(\tau)$, if we consider successive measurements in $\mathrm{N}$ number of steps which consists of free evolution for a time $\tau$ plus instantaneous measurements of the observable $O$ at times $\tau_{k}=k \tau, k=1,2, \ldots . N$, then the probability of finding the system in the initial state would be given by

$$
P\left(\tau_{N}\right)=[P(\tau)]^{N}=\left(1-\tau^{2} v(0)^{2} / 4\right)^{N}
$$

For large number of measurements we can approximate the survival (inhibition) probability $P\left(\tau_{N}\right)$ as

$$
P\left(\tau_{N}\right)=e^{-\tau^{2} v(0)^{2} / 4 N}=e^{-T^{2} v(0)^{2} / 4 N}
$$

This shows that the survival probability after $N$ steps of measurement process is a geometric quantity in nature. Therefore, in the limit $N \rightarrow \infty$ the survival probability tends to unity. This leads to the QZE for systems governed by more general dynamical equations (refered to as non-Schrödinger) within the collapse hypothesis. Only requirement necessary is that the solutions of these equations are square integrable functions and thus they belong to their respective Hilbert spaces. This is always the case for the Schrödinger equation and hence it seems that Schrödinger equation is not a necessary but only a sufficient requirement for predicting QZE. Any other evolution equation, which could be non-linear, non-unitary and inhomogeneous would also predict QZE as it is related to the geometry of the Hilbert space involved.

It is interesting to investigate the meaning of $v(0)$ in the special case of linear, unitary Schrödinger time evolution of the quantum system. For Schrödinger quantum evolution, with a time-independent Hamiltonian $H$ the quantity $<\dot{\chi}(0) \mid \dot{\chi}(0)>$ goes over to $-\frac{1}{\hbar^{2}}<\Psi(0)\left|H^{2}\right| \Psi(0)>=-\frac{1}{\hbar^{2}}<\Psi(t)\left|H^{2}\right| \Psi(t)>$ and $i<\chi(0) \mid \dot{\chi}(0)>$ goes over to $\frac{1}{\hbar}<\Psi(0)|H| \Psi(0)>=\frac{1}{\hbar}<\Psi(t)|H| \Psi(t)>$. Therefore, in this case the quantity $v(0)$ is proportional to the uncertainty $\Delta E$ in the energy of the system, i.e., $v=2 \frac{\Delta E}{\hbar}$. In other words, for Schrödinger time evolution the speed of the system point on $\mathcal{P}$ is decided by the fluctuation in the energy of the system and one recovers the standard Zeno type result for survival probability, given by $P\left(\tau_{N}\right)=\exp \left(-\frac{T^{2} \Delta E^{2}}{N}\right)$, 11 which in the ultimate limit $N \rightarrow \infty$ approaches unity. 
Next, we give a criterion for the QZE to occur with finite number of measurements (since we are not taking an ideal limit $N \rightarrow \infty$ ). For a finite number $N$ of measurements, it follows from Eq.(11) that

$$
d P\left(\tau_{n}\right) / d N>0 .
$$

This may be taken as a criterion for the occurance of QZE. The geometrical nature of the survival probability $P\left(\tau_{N}\right)$ along with the condition (13) implies that for the QZE to occur, it is necessary that the dynamics of a quantum system is characterised by an evolution equation whose solutions spans a Hilbert space. In essence the evolution of a quantum system can be quite arbitrary between two successive measurements and QZE follows purely from the collapse of the wavefunction and the geometry of the Hilbert space.

Using these ideas we illustrate how can one observe the quantum Zeno effect for system described by non-linear and non-unitary equations. As a first example we consider the non-linear Schrödinger equation

$$
i \hbar \frac{\partial \Psi(\mathbf{x}, t)}{\partial t}=-\frac{\hbar^{2}}{2 m} \nabla^{2} \Psi(\mathbf{x}, t)+V(\mathbf{x}) \Psi(\mathbf{x}, t)-b|\Psi|^{2} \Psi(\mathbf{x}, t)
$$

where $V(\mathbf{x})$ is the confining potential and $b$ is the non-linearity parameter whose meaning depends on the problem considered. This equation has been used in various contexts. It describes the dynamics of quasi-particles in condensed matter physics. More recently, this equation has been of much interest in understanding the behaviour of Bose-Einstein condensates in the confining trap potential [21].

This equation preserves the norm of the wavefunction, that is, $\|\dot{\Psi}\|=0$ for all times. We will show that a system described by Eq(14) conforms to the short time behaviour as given by Eq.(5). As a particular case, we consider a one-dimensional form of Eq.(14) with $V(x)=0$, written in the form

$$
i \hbar \frac{\partial \Psi(x, t)}{\partial t}+\frac{\hbar^{2}}{2 m} \frac{\partial^{2} \Psi(x, t)}{\partial x^{2}}+b|\Psi|^{2} \Psi(x, t)=0
$$

where we assume that $b>0$. This equation admits a propagating soliton-like solution given by

$$
\Psi(x, t)=\left(\frac{a}{b}\right)^{\frac{1}{2}} \eta e^{-i(\omega t-u x / a)} \operatorname{sech}[\eta(x-u t)]
$$

where $\eta=\frac{1}{a}\left(u^{2}-2 a \omega\right)^{\frac{1}{2}}, a=\frac{\hbar^{2}}{m}$. It is easy to check that $\|\Psi\|^{2}=(2 \eta a / b)$ which shows that the norm of the wavefunction does not change with time. Using Eq.(11) we can see that the speed at which the system point move on the projective Hilbert space is given by $v^{2}=$ $4(\eta u)^{2} / 3$. The short time survival probability is given by $P(\tau)=\left(1-\tau^{2} \eta^{2} u^{2} / 3\right)$. Therefore, the survival probability after $\mathrm{N}$ steps of measurement is $P\left(\tau_{N}\right)=\exp \left(-\eta^{2} u^{2} T^{2} / 3 N\right)$ which 
satisfies (13) and hence the QZE occurs. It is interesting to note that the Zeno effect in this case would imply an inhibition of the propagation of the solitonic wave.

A second example is provided by a model proposed by Gisin [22] for description of quantum dissipative systems. This phenomenological non-linear equation reads as

$$
i \hbar \frac{\partial \mid \Psi(t)>}{\partial t}=H\left|\Psi(t)>+i \lambda\left(\frac{<\Psi|H| \Psi>}{<\Psi \mid \Psi>}-H\right)\right| \Psi(t)>
$$

where $H$ is the Hamiltonian of the system and $\lambda \geq 0$ is a dimensionless damping constant. This equation also preserves the norm of the state vector during its evolution. Another interesting feature is that this equation retains most of the conventional interpretation of the quantum theory.

We apply Gisin's equation to a two-level system. Let us consider a simple case of the model of a two-level atom interacting continuously with a coherent field. The Hamiltonian of the system is given by

$$
H=\hbar \omega S_{+} S_{-}-\frac{\hbar \alpha}{2}\left(S_{+} e^{-i \omega t}+S_{-} e^{i \omega t}\right)
$$

where $S_{+}=|g><e|, S_{-}=|e><g| ;|g>| e>$, being the "ground" and "excited" states of the atom and $\alpha$, the Rabi frequency related to the amplitude of the driving field. For simplicity, we ignore the spontaneous emission from the excited state and also assume that the field is in resonance with the atomic transition frequency $\omega$. The general state of the system belongs to a Hilbert space of dimension two and is given by

$$
|\Psi(t)>=a(t)| g>_{+} b(t) e^{-i \omega t} \mid e>
$$

and inserting (18) and (19) in Gisin's equation (17), we obtain

$$
\begin{aligned}
& \dot{a}(t)=\frac{\alpha}{2}(\lambda+i) b(t)+\lambda\left[\omega|b(t)|^{2}-\frac{\alpha}{2}\left(a^{*}(t) b(t)+a(t) b^{*}(t)\right)\right] a(t) \\
& \dot{b}(t)=\frac{\alpha}{2}(\lambda+i) a(t)+\lambda\left[\omega\left(|b(t)|^{2}-1\right)-\frac{\alpha}{2}\left(a^{*}(t) b(t)+a(t) b^{*}(t)\right)\right] b(t) .
\end{aligned}
$$

It is easy to obtain the short-time behaviour of the solutions of the above equations. Thus, if we assume that the atom is initially in the ground state $\mid g>$, then the survival probability $P(\tau)$ is given by

$$
P(\tau)=1-\frac{\alpha^{2}}{4}\left(\lambda^{2}+1\right) \tau^{2}
$$

The system point moves on the projective Hilbert space (which is a sphere $S^{2}$ for twolevel system) of the quantum system with a speed $v=\alpha\left(\lambda^{2}+1\right)^{1 / 2}$ Also, we can calculate the Fubini-Study metric during the time $T$ which is given by $s=\alpha\left(\lambda^{2}+1\right)^{1 / 2} T$ Therefore, with the increase of number of measurements the survival probability approaches $P\left(\tau_{N}\right)=$ 
$\exp \left(-\frac{\alpha^{2}}{4}\left(\lambda^{2}+1\right) T^{2} / N\right)=\exp \left(-s^{2} / 4 N\right)$, and the quantum Zeno effect in this case implies that the atom remains in the ground state. It may be interesting to note that the presence of the damping constant $\lambda$ in the survival probability enhances the quantum Zeno effect.

In conclusion, we have shown in this paper that the short time quadratic behaviour of the transition probability is a natural consequence of the inner product of the Hilbert

space, using which the former is defined. The survival probability is related to the speed of transportaion of the system point on the projective Hilbert space of the quantum system. It is shown that the Schrödinger time evolution between two successive measurements is not a necessary but only a sufficient condition to predict quantum Zeno effect. Any non-linear, non-unitary and inhomogeneous evolution equation could also predict quantum Zeno effect within the von Neumann's collapse postulate. More importantly, it seems that the presence of non-linear and damping parameters in the system can enhance the Zeno process with a finite number of measurement pulses. It would indeed be interesting to study the role of non-linearity and damping parameters in real quantum Zeno type experiments in future.

\section{ACKNOWLEDGEMENTS}

The authors wish to thank Prof. E. C. G. Sudarshan for some stimulating discussions on the subject matter of Zeno effect for non-linear quantum systems. 


\section{REFERENCES}

[1] B. Mishra and E. C. G. Sudarshan, J. Math. Phys. 18 (1977) 756.

[2] V. B. Braginsky and E. Ya. Khalily, Quantum measurement, ed. K. S. Thorne (Cambridge Univ. Press, Cambridge, 1992);

[3] J. von Neumann, Matematische Grundlagen der Quantenmechanik (Berlin, 1932).

[4] W. H. Itano et al Phys. Rev. A 41 (1990) 2295.

[5] R. J. Cook, Phys. Scr. T 21 (1988) 49.

[6] L. E. ballentine, Phys. Rev. A 43 (1991) 5165.

[7] T. Petrovsky, S. Tasaki and I. Prigogine, Phys. Lett. A 151 (1990) 109; A. Peres and A. Ron, Phys. Rev. A 42 (1990) 5720; S. Pascazio, M. Namiki, G. Badurek and H. Rauch, Phys. Lett. A 1993) 155; D. Home and M. A. B. Whitaker, J. Phys. A 25 (1992) 657.

[8] G. C. Ghiradi, C. Omero, T. Weber and A. Rimini, Nuovo Cimento 52 A (1979) 421.

[9] H. Nakazato, M. Namiki, S. Pascazio and H. Rauch, Phys. Lett. A 199 (1995) 27.

[10] A. Venugopalan and R. Ghosh, Phys. Lett. A. 204 (1995) 11.

[11] A. K. Pati, Phys. Lett. A 215 (1996) 7.

[12] C. Presilla, R. Onofrio and U. Tambini, Ann. Phys. 248 (1996) 95; (also references there in).

[13] M. B. Mensky, R. Onofrio and C. Persilla, Phys. Rev. Lett. 70 (1993) 2825.

[14] W. L. Power and P. L. Knight, Phys. Rev. A 53 (1996) 1052.

[15] A. Schenzle, Contemp. Phys., 37 (1996) 303.

[16] D. Home and M. A. B. Whitaker, Ann. Phys. 258 (1997) 237.

[17] J. P. Provost and G. Vallee, Commun. Math. Phys. 76 (1980) 289.

[18] J. Anandan and Y. Aharonov, Phys. Rev. Lett. 65 (1990) 455.

[19] A. K. Pati, Phys. Lett. A 159 (1991) 105.

[20] A. K. Pati, Phys. Lett. A 202 (1995) 40.

[21] S. Stringari, Phys. Rev. Lett. 76 (1996) 1405; M. Edwards et al, Phys. Rev. Lett. 77 (1996) 1671.

[22] N. Gisin, J. Phys. A 14 (1981) 2259; Physica A 111 (1982) 364.

[23] See for example a Review article by J. Anandan, Nature, 360 (1996) 307.

[24] A. K. Pati and S. V. Lawande, Phys. Lett. A 223 (1996) 233. 